\title{
Fisiología y bioquímica de la maduración del fruto de durazno [Prunus persica (L.) Batsch]. Una Revisión
}

\section{Physiology and biochemistry of peach [Prunus persica (L.) Batsch] fruit maturation. A review}
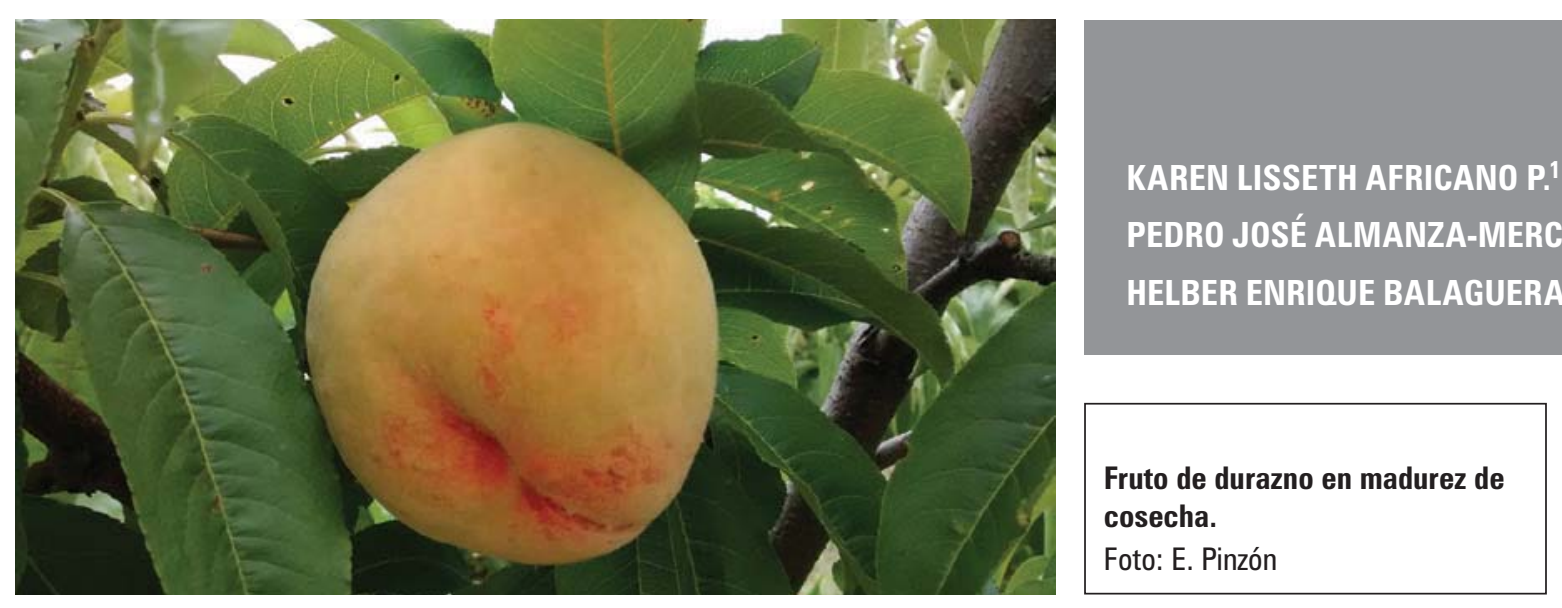

\section{RESUMEN}

El durazno pertenece a la familia Rosaceae; es una drupa muy apetecida por su agradable sabor y cualidades nutricionales, al igual que en la industria de alimentos. Es un fruto climatérico, altamente perecedero con altas tasas respiratorias y de producción de etileno. Durante la maduración presenta pérdida del color verde de la epidermis y adquiere tonalidades rojizas/amarillas, por degradación de clorofilas y biosíntesis de antocianinas y carotenoides; también presenta aumento en el contenido de azúcares solubles, emisión de compuestos volátiles y actividad antioxidante, disminución en el contenido de ácidos orgánicos, firmeza y almidón. Debido a las características fisiológicas y bioquímicas propias del fruto relacionadas con la alta perecibilidad, se dificultan las labores de manipulación, transporte y comercialización, por lo anterior se realizó la actualización de la fisiología y bioquímica de la maduración del fruto de durazno.

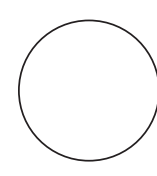

Palabras clave adicionales: cambios fisiológicos, cambios bioquímicos, producción de etileno, comportamiento climatérico.

Facultad de Ciencias Agropecuarias, Programa de Maestría en Fisiología Vegetal, Universidad Pedagógica y Tecnología de Colombia (UPTC), Tunja (Colombia).

2 Facultad de Ciencias Agropecuarias, Universidad Pedagógica y Tecnológica de Colombia (UPTC), Tunja (Colombia).

3 Facultad de Ciencias, Universidad El Bosque, Bogotá (Colombia).

4 Autor para correspondencia.hbalaguera@unbosque.edu.co 


\section{ABSTRACT}

The peach belongs to the Rosaceae family. It is a drupe that is highly desired due to its pleasant taste and nutraceutical properties, such as in the food industry. The peach is a climacteric fruit that is highly perishable with high respiratory rates and ethylene production. During maturation, the peach displays a loss of green color in the peel skin and obtains reddish/yellow hues through degradation of chlorophyll and synthesis of anthocyanin and carotenoids; also, it increases the content of soluble sugars, emission of volatile compounds and antioxidant activity and decreases the content of organic acids, along with a loss of firmness and starch. Because of the physiological and biochemical characteristics that are typical for this fruit, which are related to high perishability, handling, transport and marketing are difficult; therefore, this updating of the physiology and biochemistry of peach fruit ripening was carried out.

Additional key words: physiological changes, physicochemical changes, ethylene production, climacteric behavior.

Fecha de recepción: 19-03-2015

Aprobado para publicación: 28-05-2015
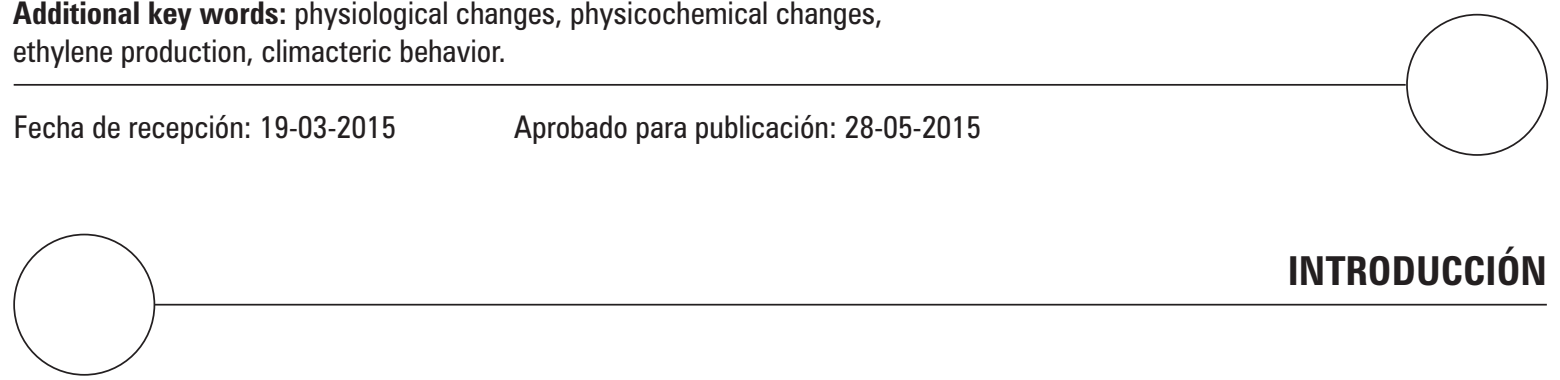

El fruto de durazno [Prunus persica (L.) Batsch, Rocaseae], es una drupa con 4 a $10 \mathrm{~cm}$ de diámetro, de colores rojizos y amarillos y forma ovoide, puede presentar exocarpo tomentoso (Cárdenas y Fischer, 2013), su peso cambia para cada variedad, por ejemplo se tiene reportes para la variedad Rubidoux en el peso, de 150 g y Dorado de $100 \mathrm{~g}$ (Chaparro et al., 2010). El duraznero es uno de los frutales comúnmente sembrados en zonas de trópico alto (o clima frío), debido a que posee ventajas comparativas con los que se siembran en las zonas templadas (Pinzón et al., 2014). El durazno es apetecido por su agradable sabor, propiedades organolépticas (Brandi et al., 2011) y propiedades medicinales. Herrera et al. (2006) indican que el consumo de este fruto aporta vitamina A, B1, B2, C, fósforo, calcio entre otros elementos y vitaminas esenciales.

El durazno es un fruto climatérico, siendo el etileno el responsable de regular los principales cambios durante la maduración, entre los que se destacan el incremento en la tasa respiratoria (Akbudak y Eris, 2004), sólidos solubles totales, cambios en el color de la epidermis y pulpa, dis- minución en la acidez total titulable y en la resistencia o firmeza de la pulpa (Altube et al., 2001). Este último es quizá el parámetro más notorio y que permite además determinar estándares de recolección, calidad y consumo, los cuales a su vez están influenciados por cambios a nivel organoléptico como es el desarrollo de sabor y aroma (Taín et al., 2011). El etileno también es responsable de acelerar la senescencia, por lo que su concentración en el fruto afecta directamente la calidad (Ramírez, 2007). El durazno es un fruto altamente perecedero, que presenta una reducida vida útil en poscosecha, comportamiento que se debe a alto porcentaje de agua (Herrera et al., 2006) y a la alta actividad metabólica que presenta el fruto, que junto con los daños mecánicos que se generan por un inadecuado transporte y almacenamiento ocasionan pérdidas poscosecha entre el 15 y 25\% (Seta y Moyano, 2007).

En la maduración, el fruto de durazno presenta un cambio en el color de fondo de la epidermis, el cual presenta una evolución de verde a rojo por degradación de clorofilas; en cuanto al color de recubrimiento este presenta un cambio de to- 
nalidades amarillo verdoso a rojo, generalmente en respuesta a la acumulación de carotenoides y antocianinas (Cunha et al., 2007). El contenido de ácidos orgánicos en durazno disminuye a medida que el fruto madura (Rodríguez-Félix et al., 2011). Durante la maduración del fruto también se llevan a cabo procesos de síntesis de compuestos volátiles, reducción del contenido de proteínas e incremento en la capacidad antioxidante del fruto (D'Ambrosio et al., 2013). El objetivo de esta revisión fue presentar y discutir los principales resultados obtenidos de investigaciones realizadas, sobre los cambios fisiológicos y bioquímicos relacionados con el proceso de maduración del fruto de durazno.

\section{PATRÓN DE RESPIRACIÓN Y PRODUCCIÓN DE ETILENO}

El durazno es catalogado como un fruto climatérico, debido a que presenta un incremento en su tasa respiratoria después de que alcanza la madurez fisiológica y se prolonga hasta la senescencia del fruto (Kader, 2002). Este aumento representativo de la tasa respiratoria durante la maduración genera cambios en la concentración de almidón y ácidos orgánicos, los cuales pueden ser utilizados como sustratos respiratorios (Akbudak y Eris 2004). De acuerdo con Brovelli et al. (1999), durante la maduración el durazno presenta una tasa respiratoria moderada con valores entre 59 a 102 mg CO $\mathrm{kg}^{-1} \mathrm{~h}^{-1}$; según Santana et al. (2011) para durazno 'Douradão' la tasa de respiración fue de $95 \mathrm{mg} \mathrm{CO} \mathrm{kg}^{-1} \mathrm{~h}^{-1}$ al inicio de la maduración, y alcanzó valores entre $120-130$ mg CO $\mathrm{kg}^{-1} \mathrm{~h}^{-1} \mathrm{a}$ medida que avanzó el proceso.

La respiración puede verse afectada por factores como la temperatura, de acuerdo con Machuca et al. (2010), frutos de durazno variedad Royal Glory mostraron tasas respiratorias de $15 \mathrm{mg}$ $\mathrm{CO}_{2} \mathrm{~kg}^{-1} \mathrm{~h}^{-1}$ cuando fueron almacenados a $4^{\circ} \mathrm{C}$, y de $20 \mathrm{mg} \mathrm{CO}_{2} \mathrm{~kg}^{-1} \mathrm{~h}^{-1}$ cuando la temperatura de almacenamiento fue de $8^{\circ} \mathrm{C}$, concluyéndose que a mayor temperatura se presentan mayores tasas de respiración. Victoria-Escamilla et al. (2013) mencionan que en frutos con algún tipo de daño físico también se presenta aumento en la intensidad respiratoria, lo cual se puede relacionar con disminución de la vida útil del fruto.

De otra parte, el etileno es la hormona encargada de regular diferentes procesos durante la maduración y senescencia en el fruto de durazno ( $\mathrm{Ra}$ mírez, 2007; Tonetto de Freitas et al., 2007; Rodríguez et al., 2011). La acción del etileno inicia cuando se une a receptores de las membranas de las células (Hernández et al., 2007), proceso que conlleva a una serie de eventos que culminan con cambios en la expresión génica. Zhang et al. (2012) indican que la presencia de etileno es indispensable para dar inicio a la maduración del fruto de durazno y está relacionado con las respuestas fisiológicas que se dan en esta fase, como cambio de color, ablandamiento de la pulpa, desarrollo de sabor, entre otros procesos asociados con la maduración y senescencia del fruto.

La concentración de etileno en el fruto de durazno es catalogada como alta, se reportan valores entre 10,0-100,0 $\mu \mathrm{L}$ de $\mathrm{C}_{2} \mathrm{H}_{4} \mathrm{~kg}^{-1} \mathrm{~h}^{-1}$ a $20^{\circ} \mathrm{C}(\mathrm{He}-$ rrera et al., 2006); de acuerdo con esto Santana et al. (2010) encontraron para duraznos 'Douradão' concentraciones de etileno de $20 \mu \mathrm{L} \mathrm{C}_{2} \mathrm{H}_{4} \mathrm{~kg}^{-1} \mathrm{~h}^{-1}$ al inicio de la maduración, mientras que en estados más avanzados la concentración aumentó hasta 50-70 $\mu \mathrm{L} \mathrm{C}_{2} \mathrm{H}_{4} \mathrm{~kg}^{-1} \mathrm{~h}^{-1}$, posteriormente disminuye en la fase de senescencia; la producción se acentuó del día 3 al 5, en los cuales se iniciaron los diferentes cambios a nivel físico, químico y fisiológico en el fruto. Por otro lado Crisosto et al. (2013) señalan que el uso de etileno exógeno en durazno genera a una maduración más uniforme del fruto, sin que se acelere la taza de maduración.

Existen compuestos que bloquean la unión del etileno a las células logrando retrasar la maduración del fruto, uno de ellos es el 1-metilciclopropeno (Balaguera-López et al., 2014). Estudios realizados por Zhang et al. (2012) permitieron corroborar que la biosíntesis de etileno es autoca- 
talítica ya que con la aplicación de 1-MCP la biosíntesis fue menor. Por otro lado investigaciones realizadas por Candan (2012); Kluge y Jacomino (2002); Dong et al. (2001) y Tonetto De Freitas et al. (2007) indican que el 1-MCP logra retrasar la maduración del fruto de durazno únicamente cuando es aplicado en estado preclimatérico.

\section{CAMBIOS EN LA FIRMEZA DEL FRUTO}

Gutiérrez et al. (2007) reportaron que la firmeza es el parámetro más usado para establecer el grado de madurez de un fruto y es un indicativo sobre su vida útil potencial. El fruto de durazno presenta un ablandamiento prematuro; este es un cambio drástico que se produce en poco tiempo y es el que genera las mayores pérdidas poscosecha, pues el debilitamiento de la estructura del fruto puede generar mayor susceptibilidad al daño mecánico y al ataque de patógenos (Di Santo et al., 2009).

Estudios realizados por Victoria-Escamilla (2013), Rodríguez-Félix (1996) y Orazem et al. (2013) muestran que la firmeza de los frutos de durazno tiende a disminuir a medida que avanza la maduración. De acuerdo con esto, Cascales et al. (2005) indican que los duraznos variedad Caterin presentaron un cambio en la firmeza de 65 a 22 Newton (N), de igual manera Ferrer et al. (2005) encontraron para duraznos cv. Calanda una disminución lineal de la firmeza con valores de $14 \mathrm{~kg} \mathrm{~cm}^{-2}$ a $4,5 \mathrm{~kg} \mathrm{~cm}^{-2}$ durante el periodo de maduración. Por otro lado, Gorny et al. (1999) reportan que el estado de madurez óptimo para duraznos y nectarinas es aquel que tiene entre 13 y $27 \mathrm{~N}$ de firmeza de la pulpa, en estos valores el fruto alcanza la vida útil máxima, y se obtiene buena calidad para consumo. Por su parte, Valero et al. (2007) clasificaron el fruto de durazno en tres clases con base en la firmeza, donde $35 \mathrm{~N}$ es la firmeza mínima que debe tener el fruto para que este no presente daño mecánico en el manejo poscosecha, frutos con 18-35 $\mathrm{N}$ son catalogados como listos para comprar, y frutos con 8-13 $\mathrm{N}$ de firmeza son aquellos listos para consumo.

En general el ablandamiento del fruto es un proceso de modificaciones en la estructura de la pared celular donde se presenta despolimerización de glicanos y solubilización de pectina (D'Ambrosio et al., 2013; Fruk et al., 2014), y donde intervienen enzimas como poligalacturonasa (PG), pectinmetilesterasa (PME), endo-1,4$\beta$-glucanasa, $\alpha$-arabinosidasa y $\beta$-galactosidasa (Rodríguez-Félix et al., 2011) entre otras. La actividad de estas enzimas durante la maduración está relacionada con un cambio en los polisacáridos de la pared celular; el tiempo y momento de actividad varía entre las enzimas y no siempre están relacionadas con el incremento de los niveles de etileno (Brummell et al., 2004). RodríguezFélix et al. (2011) determinaron que en la maduración de frutos de durazno cv. Flordaprince la actividad de PME es mayor al inicio y disminuye a medida que avanza la maduración, mientras que la actividad de PG aumenta con la maduración, coincidiendo con la pérdida de firmeza de los frutos. Manganaris et al. (2007) obtuvieron el mismo comportamiento para estas enzimas en frutos cv. Andross, además determinaron que la pérdida de firmeza de los frutos se retrasó con aplicaciones de cloruro de calcio.

En durazno el ablandamiento que se da en forma prematura ocurre cuando la pulpa del fruto está en la transición de color verde a amarillo, antes del pico del etileno y antes de que inicie la maduración, y aumenta rápidamente cuando se genera el cambio de color del mesocarpio y a medida que el contenido de etileno incrementa (Brummell et al., 2004). Hayama et al. (2006) determinaron que frutos con aplicación de etileno en el rango de 0,1-100 $\mu \mathrm{L} \mathrm{L}^{-1}$ mostraron ablandamiento, mientras que los frutos que no se trataron con etileno no mostraron reducciones notables en su firmeza, esto les permitió concluir que la presencia y concentración de etileno es indispensable para la iniciación, avance y regulación de la tasa de ablandamiento de los frutos. 


\section{CAMBIOS ASOCIADOS AL COLOR}

En durazno el color es uno de parámetros más usados como índice de madurez no destructivo, por medio del cual se puede determinar el momento óptimo de cosecha, y cuyo valor «a» de Hunter debe estar en el rango de - 5,8 y - $-1,3$ según los estándares de madurez mínima (Budde et al., 2003). Crisosto et al. (2013) indican que aunque el momento de cosecha está determinado por el cambio de color de fondo de la piel, de verde a rojizo, y por la firmeza de la pulpa; estos varían entre las diferentes variedades, haciendo difícil generalizar un punto óptimo de cosecha.

Rodríguez-Félix et al. (2011) encontraron en frutos de duraznos variedad 'Flordaprince', cosechados en cinco estados de madurez con base en el color de la epidermis, que a medida que avanzó la maduración, el ángulo del tono disminuyó significativamente, lo que indica que los valores más altos son para los frutos verdes y los más bajos para frutos con tonalidades amarillas o amarillo-rojizas, los valores de claridad de la epidermis también disminuyeron significativamente, esto indica que los colores al final de la maduración son más oscuros; por otro lado el croma aumentó para esta variedad, lo que significa que a medida que avanza la maduración se presenta mayor saturación del color.

Por su parte, los estudios realizados por Orazem et al. (2013) permitieron evidenciar un cambio de color en el fruto de durazno en la fase de maduración, en la cual los valores de $\mathrm{L}^{*}$ disminuyeron significativamente provocando un oscurecimiento del fruto y un aumento de tonalidades rojizas, esto también se correlaciona con un cambio en el valor a* de negativo a positivo. Cunha et al. (2007) encontraron en durazno var. Aurora-1 durante la maduración que el color de fondo presenta un cambio de verde a amarillo como resultado de la degradación de clorofila por enzimas como clorofilasas, síntesis de carotenoides o por modificaciones en el pH; el color de recubrimiento también presenta un cambio de color muy marcado de amarillo verdoso a rojo, ya que hay una alta síntesis y acumulación de antocianinas y carotenoides.

La coloración del fruto de durazno depende de pigmentos como antocianinas y carotenoides, se destacan los siguientes: fitoenos, $\alpha$ - y $B$-carotenos, $\gamma$-caroteno, luteína, zeaxantina, entre otros, estos son los responsables del color amarillo-rojo del fruto (Herrero y Guardia, 1992). En correlación, a medida que el proceso de maduración avanza, los contenidos de clorofila disminuyen de manera significativa, mientras que el contenido de carotenoides y antocianinas aumenta (Cascales et al., 2005), en estudios realizados por Brandi et al. (2011) en duraznos 'Redhaven', la acumulación máxima de carotenoides se logró a los 122 d después de la polinización, es decir al final de la madurez. Según Ferrer et al. (2005) las enzimas oxidativas polifenoloxidasa (PPO) y peroxidasa (POD) están involucradas en el cambio de color del fruto, ya que estas promueven reacciones y cambios estructurales que conllevan al pardeamiento y pérdida del color, este último en frutos cosechados.

\section{CAMBIOS EN AZÚCARES SOLUBLES Y ÁCIDOS ORGÁNICOS}

En la maduración los cambios a nivel de carbohidratos se derivan de la conversión del almidón en azúcares solubles, incrementándose el sabor dulce en los frutos, también hay degradación de carbohidratos poliméricos, almidón y celulosa, por lo que además del sabor, se ve afectada la textura del fruto (Hiwasa y Ezura, 2014). Los azúcares presentes en el fruto de durazno son sacarosa, glucosa, fructosa y en menores cantidades sorbitol (Cantín et al., 2009a) e inositol, este último con una concentración del 1,1\% (Moriguchi et al., 2007) los frutos maduros presentan un alto contenido de sacarosa 50-75\% (Byrne et al., 1991), por lo que es considerado el azúcar predominante en este fruto (Desnoues et al., 2014); estudios realizados por Borsani et al. (2009) en durazno cv. Dixiland mostraron una disminución significativa en el contenido de este azúcar 
en el día 7 después de la cosecha con un contenido aproximado de $2,5 \mathrm{~g} / 100 \mathrm{~g}$ de peso fresco del fruto, asimismo para el día 7 después de la cosecha se presentó un aumento significativo en el contenido de glucosa y fructosa con valores aproximados de 0,96 y 0,97 g/100 g de peso fresco; mientras que el contenido de sorbitol solo fue alto en el momento de la cosecha $(0,22 \mathrm{~g} / 100 \mathrm{~g}$ de peso fresco), sin embargo este contenido es bajo comparado con el de glucosa, sacarosa y fructosa. De otra parte, Victoria-Escamilla et al. (2013) indican que en la poscosecha el fruto presenta pérdidas a nivel de calidad, valor nutricional y propiedades organolépticas como resultado de la degradación de azúcares y otros compuestos orgánicos en la respiración.

Existen varios factores que pueden afectar el contenido de carbohidratos en el fruto de durazno, como el estado de madurez en la cosecha (Herrera et al., 2006), y prácticas agrícolas como el raleo. Casierra-Posada et al. (2007) encontraron que los sólidos solubles totales fueron mayores cuando en el raleo se dejaron 40 y 50 hojas por fruto, debido a que con esta práctica se afecta la relación fuente-vertedero y Fischer et al. (2012) afirman que una calidad alta del fruto requiere una relación adecuada entre hojas y fruto (número de hojas, área foliar por fruto o unidad peso seco del fruto).

Por otro lado, los ácidos orgánicos predominantes en el durazno son ácido málico, ácido cítrico y ácido quínico; estos disminuyen a medida que el fruto madura (Rodríguez-Félix et al., 2011) como mencionan Day et al. (1997) y Wu et al. (2003); los ácidos orgánicos son usados como sustratos respiratorios por el fruto, y además pueden ser convertidos en azúcares; de esta manera la concentración inicial de azúcares debe ser menor al inicio de la poscosecha. En cuanto al comportamiento de los ácidos orgánicos en frutos de durazno 'Dixiland' se presentó un aumento significativo en la concentración de ácido cítrico en el día 7 después de la cosecha con una concentración de $0,4 \mathrm{~g} \mathrm{~g}^{-1}$ de peso fresco, mientras que el ácido málico no mostró cambios significativos (Borsani et al., 2009).
En el fruto de durazno el contenido de ácido ascórbico (vitamina C) está aproximadamente entre 1 y $14 \mathrm{mg} / 100 \mathrm{~g}$ de peso fresco para la pulpa (Tavarini et al., 2008), sin embargo su contenido es mayor en la epidermis (Gil et al., 2002), ya que este es usado por el fruto como protección al estrés generado por la luz (Zhishen et al., 1999); el contenido de vitamina C es dependiente principalmente del genotipo, aunque también está relacionado con prácticas culturales como la frecuencia de riego (Lee y Kader, 2000), la fertilización nitrogenada, y con factores ambientales como la radiación y la temperatura. Otro factor importante que también puede afectar el contenido de vitamina $\mathrm{C}$ es el estado de madurez al momento de la cosecha (Solarte et al., 2010). Cantín et al. (2009b) obtuvieron diferentes contenidos de vitamina C en frutos de pulpa blanca $(4,8 \mathrm{mg} / 100 \mathrm{~g}$ de peso fresco) y de pulpa amarilla $(3,5 \mathrm{mg} / 100 \mathrm{~g}$ de peso fresco); los cuales se encuentran dentro del rango mencionado anteriormente.

\section{ACTIVIDAD ANTIOXIDANTE}

Según Saltmarsh et al. (2003) los frutos son una fuente importante de vitaminas, minerales $y$ compuestos antioxidantes, estos compuestos ayudan a reducir el riesgo de enfermedades crónicas (Buttriss, 2012). Una de las principales razones que explica la gran aceptación de los frutos de durazno entre los consumidores, está relacionada con sus propiedades organolépticas, nutricionales (Brandi et al., 2011) y medicinales (Morales, 2011). Araya et al. (2006) reportan que el fruto de durazno tiene una capacidad antioxidante baja correspondiente a 0,050 $\pm 0,010$ mmoles Fe/100 g (promedio 土desviación estándar) determinado por el método FRAP; sin embargo debido a su producción y su frecuencia de consumo, representa una fuente importante de compuestos antioxidantes (Derail et al., 1999). Por otro lado Zapata et al. (2014) indican que el fruto de durazno presenta bajo contenido de fenoles totales ( $<100 \mathrm{mg} / 100 \mathrm{~g}$ ), pero alta capa- 
cidad antioxidante (>5.000 $\mu \mathrm{mol}$ trolox/100 g), la cual se puede atribuir a la presencia de otros compuestos no fenólicos como los carotenoides. García et al. (2011) evaluaron la capacidad antioxidante de frutos de durazno cv. Prisco por el método ORAC obteniendo 18,15 mmol TE/100 $\mathrm{g}$ peso fresco y $2,5 \mathrm{mmol} \mathrm{Fe} / 100 \mathrm{~g}$ mediante el método FRAP. De acuerdo con Cantín et al. (2009b) el genotipo, las condiciones climáticas o portainjerto son determinantes de la capacidad antioxidante, la cual aumenta con la maduración del fruto (D`Ambrosio et al., 2003).

La actividad antioxidante del fruto de durazno también depende de los carotenoides (Carranco et al., 2011), y de los compuestos fenólicos como antocianinas (Garzón, 2008); en la maduración suele haber mayor biosíntesis de estos compuestos, por lo que su contenido incrementa (Orazem et al., 2013). Asimismo la actividad de los carotenoides implica la inactivación de especies reactivas de oxígeno, mientras que la función de las antocianinas está relacionada con una protección contra los daños oxidativos (Viñas et al., 2013). En el fruto de durazno, la participación de las antocianinas en la capacidad antioxidante total del fruto es mayor que la participación de los carotenos (Gil et al., 2002). De acuerdo con García et al. (2011), la concentración de antocianinas pueden verse afectados por la variedad del fruto, su estado de madurez y la fertilización del cultivo.

Los carotenoides $\beta$-criptoxantina y $\beta$-caroteno son provitaminas $A$, es decir que después de ser consumidos se pueden convertir en vitamina $A$ (Maulén et al., 2012). Pamplona (2003) indica que el contenido de vitaminas A, C y E del fruto de durazno le confieren un efecto antioxidante de gran importancia para la conservación y funcionamiento de las arterias coronarias, por lo cual su consumo beneficia el sistema cardiaco en general. La luteína y zeaxantina presentes en este fruto cumplen un papel importante en la visión, como reportan Sasaki et al. (2012), en donde la luteína actúa contra el estrés oxidativo generado por la luz en la retina. Otros beneficios que se obtienen mediante el consumo del durazno, es el aportado por el sorbitol que contribuye a la salud dental; y por la sacarosa y fructosa sobre la salud gastrointestinal (Font et al., 2013).

\section{EMISIÓN DE COMPUESTOS VOLÁTILES}

En el fruto de durazno, el sabor junto con el aroma son de las características más importantes que se relacionan con la calidad (Visai y Vanoli, 1997), siendo las lactonas $\gamma$ - y $\delta$-decalactona, las principales responsables del aroma en este fruto, al asociarse a otros compuestos volátiles como terpenos, alcoholes y aldehídos (Jia et al., 2005).

Por otro lado, Brandi et al. (2011) indican que los carotenoides son muy importantes no solo por su participación en la fotosíntesis como pigmentos accesorios, sino que además participan en la determinación de aromas ya que por acción de enzimas dioxigenasas (Rosati et al., 2009) se generan compuestos volátiles como $\mathrm{C}_{13}$-norisoprenoides, también llamados apocarotenoides; de acuerdo con Winterhalter y Schreier (1994) estos compuestos tienen un gran impacto en el aroma de frutos y flores aun estando en niveles bajos, debido a que presentan un bajo umbral de olor; sus niveles aumentan durante la maduración del fruto de durazno (Aubert et al., 2003), otros compuestos volátiles encontrados en este fruto variedad Redhaven son furano, lactona y monoterpenos (Brandi et al., 2011); de igual manera Maulén et al. (2012) identificaron en durazno variedad Royal Glory a hexanal y acetato de etilo como compuestos representativos del perfil aromático de estos frutos, este último presenta un aumento en sus niveles de concentración durante el proceso de maduración del fruto. Por su parte Guillot et al. (2006) identificó en albaricoque a $\gamma$-decalactona, acetato de etilo, acetato de hexilo, $\beta$-ionona, como los principales compuestos responsables del aroma en este fruto. En cuanto a la distribución de los compuestos volátiles Aubert y Milhet (2007) determinaron que para durazno cv. Maura, un fruto de pulpa 
blanda, los niveles superiores de compuestos volátiles se presentaron en la epidermis.

\section{CONCLUSIONES}

En las diferentes investigaciones mostradas en la revisión se encuentra que el fruto de durazno presenta alta perecibilidad asociada entre otros factores a su carácter climatérico y alta producción de etileno. Los diferentes estudios muestran que la relación tasa respiratoria-temperatura es importante para el manejo poscosecha del producto; donde el almacenamiento a baja temperatura conlleva a la disminución de la tasa respiratoria y finalmente a una reducción de la actividad metabólica en el fruto.

El fruto de durazno durante la maduración presenta un rápido ablandamiento de la epidermis, lo cual limita su manejo poscosecha y posterior comercialización. El contenido de carbohidratos presenta una dinámica importante durante esta fase, que se caracteriza la degradación hidrolítica del almidón de reserva, y su conversión en azúcares solubles. Durante la maduración se presentan cambios importantes en el color, en el contenido de clorofilas, de carotenoides y antocianinas. Estas características son determinantes para la madurez fisiológica y de consumo, y desempeñan un papel importante en la comercialización del producto. Es de destacar que durante la maduración hay un aumento en la síntesis de compuestos volátiles que aportan al aroma característica del fruto; aspecto en el cual se debe investigar con el fin de establecer su relación con la madurez fisiológica y de consumo.

A pesar del conocimiento acerca de la fisiología y bioquímica de la maduración del fruto de durazno, es importante realizar estudios moleculares para entender con mayor profundidad las causas de su rápido ablandamiento, resultados que finalmente conduzcan a mantener la calidad y prolongar la vida útil del fruto de durazno.

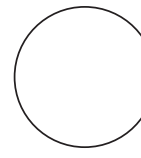

Akbudak, B. y A. Eris. 2004. Physical and chemical changes in peaches and nectarines during the modified atmosphere storage. Food Control 15, 307313. Doi: 10.1016/S0956-7135(03)00082-3

Altube, H., C. Budde, M. Ontivero y R. Rivata. 2001. Determinación de los índices de cosecha de duraznos cvs. Flordaking y San Pedro 16-33. Agricultura Técnica. 61(2), 140-150. Doi: http://dx.doi. org/10.4067/S0365-28072001000200004

Araya, H. y C. Clavijo y C. Herrera. 2006. Capacidad antioxidante de frutas y verduras cultivados en Chile. Arch. Latinoam. Nutr. 56(4), 361-365.

Aubert, C., Z. Günata, C. Ambid y R. Baumes. 2003. Changes in physicochemical characteristics and volatile constituents of yellow- and whitefleshed nectarines during maturation and artificial ripening. J. Agr. Food Chem. 51(10), 3083-3091. Doi: 10.1021/jf026153i

\section{REFERENCIAS BIBLIOGRÁFICAS}

Aubert, C. y C. Milhet. 2007. Distribution of the volatile compounds in the different parts of a white-fleshed peach (Prunus persica L. Batsch). Food Chem. 102, 375-384. Doi:10.1016/j.foodchem.2006.05.030

Balaguera-López, E., F. Salamanca-Gutiérrez, J. García y A. Herrera-Arévalo. 2014. Etileno y retardantes en la poscosecha de productos agrícolas. Una revisión. Rev. Colomb. Cienc. Hortic. 8(2), 302-313. Doi: $10.17584 /$ rcch.v8i2.3222

Borsani, J., C. Budde, L. Porrini, M. Lauxmann, V. Lombardo, R. Murray, C. Andreo, M. Drincovich y M. Lara. 2009. Carbon metabolism of peach fruit after harvest: changes in enzymes involved in organic acid and sugar level modifications. J. Exp. Bot. 60(6), 1823-1837. Doi: 10.1093/jxb/erp055

Brandi, F., E. Bar, F. Mourgues, G. Horvath, E. Turcsi, G. Giuliano, A. Liverani, S. Tartarini, E. Lewinsohn y C. Rosati. 2011. Study of 'Redhaven' peach 
and its white-fleshed mutant suggests a key role of CCD4 carotenoid dioxygenase in carotenoid and norisoprenoid volatile metabolism. BMC Plant Biol. 11:24. Doi: 10.1186/1471-2229-11-24

Brovelli, E., J. Brecht, W. Sherman y C. Sims. 1999. Nonmelting-flesh trait in peaches is not related to low ethylene production rates. HortScience 34(2), 313-315.

Brummell, D., V. Dal Cin, C. Crisosto y J. Labavitch. 2004. Cell wall metabolism during maturation, ripening and senescence of peach fruit. J. Exp. Bot. 55(405), 2029-2039. Doi: 10.1093/jxb/erh227

Budde, C., A. Tula, G. Polenta, C. Lucangeli y R. Murray. 2003. El color de fondo y la dureza como estimadores no destructivos de la firmeza de la pulpa en duraznos. Rev. Iberoamer. Tecnol. Postcosecha 5(2), 134-139.

Buttriss, J. 2012. Plant foods and health. pp. 1-51. En: Salter, A., H. Wiseman y G. Tucker (eds.). Phytonutrients. Blackwell Publishing Ltd., Oxford, UK.

Byrne, D., A. Nikolic y E. Burns. 1991. Variability in sugars, acids, firmness, and colour characteristics of 12 peach genotypes. J. Amer. Soc. Hort. Sci. 116(6), 1004-1006.

Candan, P. 2012. Productos inhibidores de la maduración: 1-Metilciclopropeno (1-MCP). En: Instituto Nacional de Tecnología Agropecuaria, INTA, http://inta.gob.ar; consulta: octubre de 2014.

Cantín, C., Y. Gogorcena y M. Moreno. 2009a. Analysis of phenotypic variation of sugar profile in different peach and nectarine [Prunus persica (L.) Batsch] breeding progenies. J. Sci. Food Agr. 89, 1909-1917. Doi: $10.1002 /$ jsfa.3672

Cantín, C., Y. Gogorcena y M. Moreno. 2009b. Evaluation of the antioxidant capacity, phenolic compounds, and vitamin $\mathrm{C}$ content of different peach and nectarine [Prunus persica (L.) Batsch] breeding progenies. J. Sci. Food Agr. 57(11), 4586-4592. Doi: $10.1021 /$ jf900385a

Cárdenas, J. y G. Fischer. 2013. Situación actual de los frutales caducifolios en el mundo y en Colombia. pp. 21-29. En: Miranda, D., G. Fischer y C. Carranza (eds.). Los frutales caducifolios en Colombia. Situación actual, sistemas de cultivo y plan de desarrollo. Sociedad Colombiana de Ciencias Hortícolas, Bogotá.

Carranco, M., M. Calvo y F. Pérez-Gil. 2011. Carotenoides y su función antioxidante: Revisión. Arch. Latinoam. Nutr. 61 (3), 233- 241.
Cascales, A., E. Costell, F. Romojaro. 2005. Effects of the degree of maturity on the chemical composition, physical characteristics and sensory attributes of peach (Prunus persica) cv. Caterin. Food Science \& Technology International. 11(5), 345352. Doi: $10.1177 / 1082013205057943$

Casierra-Posada, F., J. Rodríguez y J. Cárdenas. 2007. La relación hoja: fruto afecta la producción, el crecimiento y la calidad del fruto en duraznero (Prunus persica L. Batsch, cv. 'Rubidoux'). Rev. Fac. Nac. Agr. Medellín 60(1), 3657-3669.

Chaparro, P., A. Mariño, E. Pinzón, L. Sánchez y J. Sleby. 2010. Árboles frutales de hoja caduca En: Universidad Pedagógica y tecnológica de Colombia. Granja experimental Tunguavita-Paipa. http://www.uptc.edu.co; consulta: septiembre de 2014

Crisosto, C., E. Mitcham y A. Kader. 2013. Nectarine \& peach: Recommendations for maintaining postharvest quality. En: UCDAVIS Postharvest Technology, http://postharvest.ucdavis.edu; consulta: noviembre de 2014.

Cunha, L., M. Berlingieri, B. Mattiuz, R. Nogueira y J. Durigan. 2007. Caracterização da curva de maturação de pêssegos 'Aurora-1', na região de Jaboticabal-Sp. Rev. Bras. Frutic. 29(3), 661-665. Doi: 10.1590/S0100-29452007000300045

D'Ambrosio, C., S. Arena, M. Rocco, F. Verrillo, G. Novi, V. Viscosi, M. Marra y A. Scaloni. 2013. Proteomic analysis of apricot fruit during ripening. J. Proteomics 78, 39-57. Doi: 10.1016/j.jprot.2012.11.008

Day, K., G. Crisosto, C. Crisosto y M. Watkins. 1997. Survey of white flesh nectarines and peaches. Fruit Var. J. 52, 184-189.

Desnoues, E., Y. Gibon, V. Baldazzi, V. Signoret, M. Génard y B. Quilot-Turion. 2014. Profiling sugar metabolism during fruit development in a peach progeny with different fructose-to-glucose ratios. BMC Plant Biol. 14, 336-348. Doi: 10.1186/ s12870-014-0336-x

Derail, C., T. Hofmann y P. Schieberle. 1999. Differences in key odorants of handmade juice of yellow-flesh peaches (Prunus persica L.) induced by the workup procedure. J. Agr. Food Chem. 47, 4742-4745.

Di Santo, C., E. Pagano y G. Sozzi. 2009. Differential expression of $\alpha$-L-arabinofuranosidase and $\alpha$-Larabinofuranosidase/ $\beta$-D-xylosidase genes during peach growth and ripening. Plant Physiol. Biochem. 47, 562-569. Doi: 10.1016/j.plaphy.2009.02.007 
Dong, L., H. Zhou, L. Sonego, A. Lers y S. Luri. 2001. Ethylene involvement in the cold storage disorder of "Flavortop" nectarine. Postharvest Biol. Technol. 23, 105-115. Doi: 10.1016/S0925-5214(01)00106-5

Ferrer, A., S. Remón, A. Negueruela y R. Oria. 2005. Changes during the ripening of the very late season Spanish peach cultivar Calanda: Feasibility of using CIELAB coordinates as maturity indices. Sci. Hortic. 105(4), 435-446. Doi: 10.1016/ j.scienta.2005.02.002.

Fischer, G., P.J. Almanza-Merchán y F. Ramírez. 2012. Source-sink relationships in fruit species. A review. Rev. Colomb. Cienc. Hortic. 6(2), 238-253. Doi: $10.17584 /$ rcch.2012v6i2.1980

Font, C., Y. Gogorcena y M.A. Moreno. 2013. Fruit sugar profile and antioxidants of peach and nectarine cultivar son almond $\times$ peach hybrid rootstocks. Sci. Hortic. 164, 563-572. Doi: 10.1016/ j.scienta.2013.10.020

Fruk, G., Z. Cemelík, T. Jemric, J. Hribar y R. Vidrih. 2014. Pectin role in woolliness development in peaches and nectarines: A review. Sci. Hortic. 180, 1-5. Doi: 10.1016/j.scienta.2014.09.042

García, J., L. De la Rosa, B. Herrera, A. González, J. López, G. González, S. Ruiz y E. Álvarez. 2011. Cuantificación de polifenoles y capacidad antioxidante en duraznos comercializados en ciudad Juárez, México. Tecnociencia Chihuahua 5(2), 67-75.

Garzón, G. 2008. Las antocianinas como colorantes naturales y compuestos bioactivos: Revisión. Acta Biol. Colomb. 13(3), 27-36.

Gil, M., F. Tomás-Barberán, B. Hess-Pierce y A. Kader. 2002. Antioxidant capacities, phenolic compounds, carotenoids, and vitamin c contents of nectarine, peach, and plum cultivars from California. J. Agr. Food Chem. 50(17), 4976-4982. Doi: 10.1021/jf020136b

Gorny, J., B. Hess-Pierce y A. Kader. 1999. Postharvest physiology and quality maintenance of fresh-cut nectarines and peaches. Acta Hort. 48(5), 173-179.

Guillot, S., L. Peytavi, S. Bureau, R. Boulanger, J.P. Lepoutre, J. Crouzet y S. Galindo-Schorr. 2006. Aroma characterization of various apricot varieties using head space solid phase microextraction combined with gas chromatography-mass spectrometry and gas chromatography-olfactometry. Food Chem. 96, 147-155. Doi: 10.1016/j.foodchem.2005.04.016

Gutiérrez, A., J. Burgos y E. Molto. 2007. Pre-commercial sorting line for peaches firmness assessment. J. Food Eng. 81, 721-727. Doi: 10.1021/jf020136b
Hayama, H., T. Shimada, H. Fujii, A. Ito y Y. Kashimura. 2006. Ethylene-regulation of fruit softening and softening-related genes in peach. J. Exp. Bot. 57(15), 4071-4077. Doi: 10.1093/jxb/erl178

Hernández, M., J. Barrera, J. Fernández-Trujillo, M. Carrillo y X. Bardales. 2007. Manual de manejo de cosecha y postcosecha de frutos de arazá (Eugenia stipitata McVaught) en la amazonia colombiana. Ed. Nomos. Bogotá.

Herrera, C., M. Laitón, A. Paredes y G. Sánchez. 2006. Manejo poscosecha en durazno y ciruelo. Corpoica; Ed. Produmedios, Bogotá.

Herrero, A. y J. Guardia. 1992. Conservación de frutos: Manual técnico. Ed. Mundi-Prensa, Madrid.

Hiwasa, K. y H. Ezura. 2014. Part I. Physiology and metabolism. pp 1-99. En: Nath, P., M. Bouzayen, J. Pech y A. Mattoo. Fruit ripening: Physiology, signalling and genomics. CABI, London, UK.

Jia, H-J., A. Araki y G. Okamoto. 2005. Influence of fruit bagging on aroma volatiles and skin coloration of 'Hakuho' peach (Prunus persica Batsch). Postharvest Biol. Technol. 35, 61-68. Doi: 10.1016/ j.postharvbio.2004.06.004

Kader, A. 2002. Postharvest technology of horticultural crops. $3^{\text {rd }}$ ed. ANR Publications, Oakland, California.

Kluge, R. y A. Jacomino. 2002. Shelf life of peaches treated whit 1-methylcyclopropene. Sci. Agric. 59(1), 69-72. Doi: http://dx.doi.org/10.1590/ S0103-90162002000100010

Lee, S. y Kader, A. 2000. Preharvest and postharvest factors influencing vitamin $\mathrm{C}$ content of horticultural crops. Postharvest Biol. Technol. 20, 207-220. Doi: 10.1016/S0925-5214(00)00133-2

Machuca, A., M. Matamoros, C. Jorquera, J. Obando, L. Luchsinger, M. Gil y V. Escalona. 2010 Efecto de atmósfera modificada en durazno variedad 'Royal Glory' mínimamente procesado en fresco. En: Centro de Estudios Postcosecha (CEPOC), http://www.cepoc.uchile.cl; consulta: octubre de 2014.

Manganaris, GA., M. Vasilakakis, G. Diamantidis e I. Mignani. 2007. The effect of postharvest calcium application on tissue calcium concentration, quality attributes, incidence of flesh browning and cell Wall physicochemical aspects of peach fruits. Food Chem. 100, 1385-1392. Doi: 10.1016/ j.foodchem.2005.11.036 
Maulén, A., J. Obando-Ulloa, G. Barraza, A. Machuca, Á. Peña-Neira, L. Luchsinger y V. Escalona. 2012. Establecimiento de la composición de la atmósfera más adecuada para comercializar duraznos 'Royal Glory' mínimamente procesados en "Cascos". Rev. Iberoamer. Tecnol. Postcosecha 13(2), 181-186.

Morales, A. 2011. Frutoterapia: El oro de mil colores. Ed. EDAF, Bogotá. pp. 46-47.

Moriguchi, T., Y. Ishizawa y T. Sanada. 2007. Differences in sugar composition in Prunus persica fruit and the classification by the principal component analysis. J. Jap. Soc. Hort. Sci. 59, 307-312. Doi: $10.2503 /$ jjshs. 59.307

Orazem, P., M. Mikulic-Petkovsek, F. Stampar y M. Hudina. 2013. Changes during the last ripening stage in pomological and biochemical parameters of the 'Redhaven' peach cultivar grafted on different rootstocks. Sci. Hortic. 160, 326-334. Doi: 10.1016/j.scienta.2013.06.016

Pamplona, J. 2003. Alimentos para el corazón: El durazno. p. 85. En: Salud por los alimentos. Editorial Safeliz, Madrid.

Pinzón, E.H., A. Cruz Morillo y G. Fischer. 2014. Aspectos fisiológicos del duraznero (Prunus persica [L.] Batsch) en el trópico alto. Una revisión. Rev. UDCA Act. Div. Cient. 17(2), 401-411.

Ramírez, F. 2007. Cosecha y acondicionamiento de frutas para la comercialización. pp. 136-149. En: Memorias Primer Simposio Colombiano sobre Producción, Agroindustria y Comercialización de Frutas Tropicales. Palmira, Colombia.

Rodríguez-Félix, A., M. Villegas-Ochoa, H. Soto-Valdez y M. Silveira-Gramont. 1996. Patrón de maduración de durazno 'Flordaprince'. Hortic. Mex. 4, 119-129.

Rodríguez-Félix, A., J. Fortiz-Hernández y M. VillegasOchoa. 2011. Cambios en enzimas pectolíticas durante la maduración del durazno 'Flordaprince'. Interciencia 36(1), 65-70.

Rosati, C., G. Diretto y G. Giuliano. 2009. Biosynthesis and engineering of carotenoids and apocarotenoids in plants: State of the art and future prospects. Biotechnol. Genet. Eng. Rev. 26, 151-174.

Santana, L., B. Benedetti y J. Sigrist. 2010. Sensory characteristics of 'Douradão' peaches submitted to modified atmosphere packaging. Rev. Bras. Frutic. 32(3), 700-708. Doi: 10.1590/ S0100-29452010005000108
Santana, L., B. Benedetti y J. Sigrist. 2011. Effects of modified atmosphere packaging on ripening of 'Douradão' peach related to pectolytic enzymes activities and chilling injury symptoms. Rev. Bras. Frutic. 33(4), 1084-1094. Doi: 10.1590/ S0100-29452011000400006

Saltmarsh, M., A.C. Crozier y B. Ratcliffe. 2003. Fruit and Vegetables. pp. 107-133. En: Goldberg, G (ed.). Plants: Diet and health: The Report of a British Nutrition Foundation Task Force. Blackwell Science Ltd., Oxford, UK.

Sasaki, M., K. Yuki, T. Kurihara, S. Miyake, K. Noda, S. Kobayashi, S. Ishida, K. Tsubota y Y. Ozawa. 2012. Biological role of lutein in the light-induced retinal degeneration. J. Nutr. Biochem. 23, 423-429. Doi: 10.1016/j.jnutbio.2011.01.006

Seta, S. y M. Moyano. 2007. Duraznos para el consumo en fresco en Santa Fe. ว̇Cómo definir su momento óptimo de cosecha?. Cátedra de Cultivos Intensivos. Revista Agromensajes 23(12), 1544-1545.

Solarte, M.E., M.S. Hernández, A.L. Morales, J.P. Fernández-Trujillo y L.M. Melgarejo. 2010. Caracterización fisiológica y bioquímica del fruto de guayaba durante la maduración. pp. 85-119. En: Morales, A.L. y L.M. Melgarejo (eds.). Desarrollo de productos funcionales promisorios a partir de la guayaba (Psidium guajava L.) para el fortalecimiento de la cadena productiva. Ed. Panamericana, Bogotá.

Tavarini, S., E. Degl'Innocenti, D. Remorini, R. Massai y L. Guidi. 2008. Preliminary characterisation of peach cultivars for their antioxidant capacity. Int. J. Food Sci. Technol. 43, 810-815. Doi: 10.1111/j.1365-2621.2007.01520.x

Taín, Y., A. García, A. Hernández y J. Pérez. 2011. Estudio de la variación del índice de color durante la conservación de la piña variedad Cayena Lisa a temperatura ambiente. Rev. Cie. Téc. Agr. 20(4), 12-16.

Tonetto De Freitas, S., C. Sautter, A.C Silveira y A. Brackmann. 2007. Evaluación del efecto de la aplicación de 1-MCP y la conservación en atmósfera controlada sobre la calidad poscosecha de duraznos en dos estados de madurez. Agrociencia 11(1), 67-72.

Valero, C., C. Crisosto y D. Slaughter. 2007. Relationship between nondestructive firmness measurements and commercially important ripening fruit stages for peaches, nectarines and plums. Postharvest Biol. Technol. 44, 248-253. Doi: 10.1016/j. postharvbio. 2006.12 .014 
Victoria-Escamilla, M., S. Chaves, Y. Mendoza, J. Molina y D. Sangerman-Jarquín. 2013. Diseño, fabricación y evaluación del prototipo de simulación de esfuerzos dinámicos en durazno (Prunus persica). Rev. Mex. Cienc. Agric. 4(6), 933-945.

Viñas, M., J. Usall, G. Echeverría, J. Graell, I. Lara y D. Recasens. 2013. Poscosecha de pera, manzana y melocotón. Mundi-Prensa Libros, Lérida, España.

Visai, C. y M. Vanoli. 1997. Volatile compound production during growth and ripening of peaches and nectarines. Sci. Hortic. 70(1), 15-24. Doi: 10.1016/S0304-4238(97)00032-0

Winterhalter, P. y P. Schreier. 1994. C13-Norisoprenoid glycosides in plant tissues: an over view on their occurrence, composition and role as flavor precursors. Flavour Fragance J. 9, 281-287. Doi: 10.1002/ffj.2730090602
Wu, B., B. Quilot, J. Kervella, M. Génard y S. Li. 2003. Analysis of genotypic variation of sugar and acid contents in peaches and nectarines through the principle component analysis. Euphytica 132, 375384. Doi: 10.1023/A:1025089809421

Zapata, S., A. Piedrahita y B. Rojano. 2014. Oxygen radical absorbance capacity (ORAC) and phenolic content of fruits and vegetables from Colombia. Perspect. Nutr. Humana 16(1), 25-36.

Zhang, L., L. Jianga, Y. Shia, H. Luoa, R. Kanga y Z. Yua. 2012. Post-harvest 1-methylcyclopropene and ethephon treatments differently modify protein profiles of peach fruit during ripening. Food Res. Int. 48, 609-619. Doi: 10.1016/j.foodres.2012.05.022

Zhishen, J., T. Mengcheng y W. Jianming. 1999. The determination of flavonoid contents in mulberry and their scavenging effects on superoxide radicals. Food Chem. 64, 555-559. Doi: 10.1016/ S0308-8146(98)00102-2 